\title{
Differentiation of Porcine Mycoplasma Strains
}

\author{
By Z. DINTER, D. DANIELSSON AND K. BAKOS \\ Institutes of Virology and Bacteriology, Uppsala University, Uppsala, \\ and State Veterinary Medical Institute, Stockholm, Srweden
}

(Received 23 March 1965)

\begin{abstract}
SUMMARY
Porcine Mycoplasma strains, previously called 'swine enzootic pneumonia strains' are antigenically closely related to Mycoplasma hyorhinis. Other porcine Mycoplasma strains which are culturally and antigenically distinct from $M$. hyorhinis are more heterogeneous and fall into four serotypes. $M$. granularum, however, is antigenically unrelated to these strains.
\end{abstract}

\section{INTRODUCTION}

Different types of Mycoplasma have been isolated from pigs with enzootic pneumonia or atrophic rhinitis in Sweden (Bakos \& Dinter, 1963). The majority of isolates were shown to be culturally closely related to a cytopathogenic agent cultivated by Wesslén \& Lannek (1954) from pigs with a disease called swine enzootic pneumonia (SEP). These isolates were hence labelled SEP strains, although no evidence was presented that such strains cause a transmissible pneumonia in pigs (Goodwin \& Whittlestone, 1964). A minor group of isolates, designated B strains, were culturally distinct from the SEP strains.

In this study the SEP and в strains have been compared with each other and with reference strains of Mycoplasma hyorhinis (Switzer, 1955) and M. granularum (Switzer, 1964) - the two hitherto described species of Mycoplasma occurring in pigs. The results show that porcine Mycoplasma strains are a heterogeneous group.

\section{METHODS}

Mycoplasma strains. Two strains of Mycoplasma hyorhinis, kindly supplied by Professor A. Freundt, University of Aarhus, Denmark, and Dr W. P. Switzer, Iowa State University of Science and Technology, Ames, Iowa, U.S.A., and one strain of $M$. granularum, kindly supplied by the latter, were investigated. The $M$. hyorhinis strains were labelled $\mathrm{F}$ and $\mathrm{s} 7$, respectively. Other strains used in this study belonged to our collection of isolates from pigs. The SEP strains labelled SEP/200, SEP/sk and $\mathrm{SEP} / \mathrm{G}$ as well as six $\mathrm{B}$ strains labelled $\mathrm{B} 1$ to $\mathrm{B} 6$ were used. The strains SEP/G and $\mathrm{B} 6$ were isolated from pig material sent by Dr H.-J. Neumann, Kiel, Germany. Stock cultures of the strains were prepared in PPLO broth, distributed in tubes in amounts of $5 \mathrm{ml}$. per tube and stored at $-60^{\circ}$ before use.

PPLO media. PPLO broth medium consisted of beef heart infusion with addition of $0.5 \%(\mathrm{w} / \mathrm{v})$ yeast extract (Difco), $0.1 \%(\mathrm{w} / \mathrm{v})$ glucose, $20 \%(\mathrm{v} / \mathrm{v})$ unheated horse serum, penicillin (100 i.u./ml.) and $1 \%(\mathrm{v} / \mathrm{v})$ of a $5 \%(\mathrm{w} / \mathrm{v})$ solution of thallium acetate. PPLO agar medium was composed of the same ingredients with addition 
of $1.2 \%(w / v)$ Special Agar-Noble (Difco). Penicillin and thallium acetate were omitted when satellite growth was required.

Viable cell count. Serial tenfold dilutions of a Mycoplasma suspension were made in PPLO broth and inoculated in amounts of $0.1 \mathrm{ml}$. on plates with PPLO agar. The plates were incubated at $37^{\circ}$ and the number of colonies counted on day 6 after inoculation.

Satellite growth. The surface of PPLO agar was first inoculated with Mycoplasma and then, along the diameter of the plates, with staphylococci according to the method of Morton, Smith \& Leberman (1949). The staphylococcus strain RC $3 \mathrm{II}$, kindly supplied by Dr J. I. Terpstra, Rotterdam, Holland, was used for this purpose.

Egg inoculation. Suspensions of Mycoplasma were inoculated into the yolk sac of chick embryos aged 5-7 days as previously described (Dinter, Wesslén \& Lannek, 1957). Pools of amniotic and allantoic fluids were used for further passages in eggs.

Cell cultures. Primary cultures of calf cells were prepared from trypsinized kidneys of newborn calves and grown at $37^{\circ}$ in tubes. Growth medium was Hanks's salt solution with $\mathbf{0 . 5} \%(\mathrm{w} / \mathrm{v})$ lactalbumin hydrolysate $(\mathrm{H}+\mathrm{Lah})$ enriched with $10 \%(\mathrm{v} / \mathrm{v})$ newborn calf serum. The maintenance medium consisted of $\mathrm{H}+\mathrm{Lah}+5 \%$ $(\mathrm{v} / \mathrm{v})$ unheated horse serum. Penicillin (100 i.u./ml.) was added to both media.

Titration of infectivity in cell cultures. Serial tenfold dilutions of a Mycoplasma suspension were made in maintenance medium and inoculated in $0.1 \mathrm{ml}$. amounts in each of 4 cell culture tubes. Four additional, non-inoculated cultures served as controls of the specificity of cell degeneration induced by Mycoplasma. All cultures were incubated at $37^{\circ}$ in upright position and in a volume of maintenance medium sufficient to cover the entire cell sheet. The results were read on day 6 or 7 after inoculation. The titres were calculated according to Kärber and are expressed as $\log _{10}$ units of TCD 50/0.1 ml.

Preparation of antigens for gel diffusion. The strains were transferred into PPLO broth by inoculating $200 \mathrm{ml}$. of this medium with $10 \mathrm{ml}$. of stock culture. A dense outgrowth was obtained after incubation for 2 or 3 days at $37^{\circ}$. The organisms were concentrated by sedimentation for $30 \mathrm{~min}$. at $35,000 \mathrm{~g}$ and $4^{\circ}$ and then washed 3 times in isotonic saline. The washed sediments were resuspended in $1 \mathrm{ml}$. of distilled water per $100 \mathrm{ml}$. of the original volume and the suspensions frozen and thawed 5 times before their use as antigens. A similar technique has previously been applied by Taylor-Robinson, Somerson, Turner \& Chanock (1963) for the preparation of antigens from human Mycoplasma strains. The antigen stocks were kept at $-20^{\circ}$.

Preparation of antisera. Antisera were prepared in rabbits with PPLO broth cultures as antigens. The Mycoplasma strains were sedimented and washed as described above. The washed sediments were resuspended in $5 \mathrm{ml}$. of isotonic saline per $100 \mathrm{ml}$. of the original volume. The suspensions were then mixed thoroughly with an equal amount of Freund's complete adjuvant (Difco). Each rabbit was given $2 \mathrm{ml}$. of the antigen-adjuvant mixture intramuscularly into each leg. After 5 or 6 weeks the rabbits were injected intravenously with $1 \mathrm{ml}$. of a suspension without adjuvant prepared as described above. Serum samples were taken 1 week after the second injection of antigen and were stored at $-20^{\circ}$. They were used unheated. 
Absorption of antisera. Antisera used for gel diffusion were absorbed with the PPLO broth medium in order to remove antibodies to the ingredients of this medium. PPLO broth was concentrated 10 times by pressure dialysis in a collodion bag (Membranfilter AG, Göttingen, Germany). Equal amounts of concentrate and antiserum were mixed and the mixture kept for $16 \mathrm{hr}$ at $4^{\circ}$. The mixture was then centrifuged for $30 \mathrm{~min}$. at $4500 \mathrm{~g}$ and $4^{\circ}$ and the supernatant concentrated to the original volume by pressure dialysis as mentioned above.

Gel diffusion. The micro modification of the Ouchterlony method (Wadsworth, 1957) was used. Tests were performed on glass plates with a $0.4 \mathrm{~mm}$. thick layer of agar gel consisting of $1 \%$ (w/v) Special Agar-Noble (Difco) in phosphate-buffered saline (PBS), pH 7.2. The wells were $3 \mathrm{~mm}$. in diameter and made in $3 \mathrm{~mm}$. thick plexiglass templates. The apertures connecting the wells with the gel were $2 \mathrm{~mm}$. wide. Templates with 3,5 or 7 wells at a distance of 5 or $6 \mathrm{~mm}$. between the edges were used. When the agar had congealed the wells were filled once with reactants. The plates were kept in a humidified atmosphere at room temperature for 2 days. The templates were then removed and the precipitation patterns recorded. The plates were washed for a few days in PBS and then dried and stained with amido black as recommended by Wadsworth (1957).

Growth inhibition. The technique was that of Clyde (1964) as applied by TaylorRobinson, Canchola, Fox \& Chanock (1964) for the differentiation of human Mycoplasma strains. The strains were cultivated in PPLO broth for 2 or 3 days at $37^{\circ}$ and the cultures then spread on PPLO agar in amounts of $0.1 \mathrm{ml}$. containing $10^{5}$ to $10^{6}$ colony-forming organisms. The PPLO agar was dried for $1 \mathrm{hr}$ at $37^{\circ}$ prior to inoculation. Filter-paper discs were soaked with $0.025 \mathrm{ml}$. of undiluted antiserum by means of wire loops invented by Takátsy (1955) and manufactured by Cooke Engineering Co., Alexandria, Virginia, U.S.A. The discs were then placed at appropriate distances from one another on the surface of PPLO agar. The results were read after incubation for 3 days at $37^{\circ}$. Zones of growth inhibition were measured as the distance between the margin of the disc and the margin of inhibition.

\section{RESULTS}

\section{Cultural characteristics of SEP strains and Mycoplasma hyorhinis}

The occurrence of different Mycoplasma strains in pigs was evident at the time of isolation (Bakos \& Dinter, 1963). The SEP strains were easier to isolate in tissue cultures (Wesslén \& Lannek, 1954) or in the yolk sac of chick embryos (Dinter et al. 1957) than on PPLO growth media. Similar results were obtained when isolating Mycoplasma hyorhinis (L'Ecuyer, Switzer \& Roberts, 1961).

A property common to SEP strains was that their growth on PPLO agar was promoted by the simultaneous cultivation of staphylococci. This phenomenon was most pronounced with SEP strains not adapted to cell-free media-the number and size of the colonies formed by SEP strains increased closer to the streak of staphylococci (Bakos \& Dinter, 1963). The strains, however, were easily adapted to cellfree media after a few passages in PPLO broth.

The adapted strains showed an extensive growth on PPLO agar. The colonies were embedded in the agar and had a granular appearance with the larger colonies showing dark centres. Smears from colonies stained with Giemsa stain (Kebo AB, 
Stockholm) showed particles with an approximate size of $0 \cdot 2 \mu$. A similar colonial structure was observed after cultivation of reference strains of Mycoplasma hyorhinis. When the method of satellite growth was applied, the colonies were enlarged adjacent to the staphylococci.

Three-day-old cultures in PPLO broth were titrated in cultures of calf kidney cells. The sEP strains and Mycoplasma hyorhinis produced cytopathic changes of the same type as described for the strains of the cytopathogenic agent isolated by Wesslén \& Lannek (1954). The titres of infectivity varied between $10^{6.25}$ and $10^{\mathbf{7 . 7 5}}$ TCD 50/0.1 ml. The SEP strains and $M$. hyorhinis proved to be lethal for chick embryos but the mortality rates were irregular as described by Dinter et al. (1957) for the cytopathogenic agent of Wesslén \& Lannek (1954).

Table 1. Differentiation of porcine Mycoplasma strains by some cultural properties

\begin{tabular}{|c|c|c|c|}
\hline Organism and strain & $\begin{array}{l}\text { Promoted by } \\
\text { satellite } \\
\text { growth }\end{array}$ & $\begin{array}{l}\text { Cytopathogenic } \\
\text { for calf } \\
\text { kidney cells }\end{array}$ & $\begin{array}{c}\text { Lethal for } \\
\text { chick embryos }\end{array}$ \\
\hline M. hyorhinis $\mathrm{F}$ & + & $+*$ & $(+) \dagger$ \\
\hline M. hyorhinis s 7 & + & + & $(+)$ \\
\hline $\operatorname{SEP} / 200$ & + & + & $(+)$ \\
\hline SEP/sk & + & + & $(+)$ \\
\hline $\mathrm{SEP} / \mathrm{G}$ & + & + & $(+)$ \\
\hline M. granularum & - & $(+) \ddagger$ & - \\
\hline B 1 & - & $(+)$ & - \\
\hline $\mathbf{B 2}$ & - & $(+)$ & - \\
\hline B 3 & - & + & $+\S$ \\
\hline B4 & - & $(+)$ & - \\
\hline B 5 & - & $(+)$ & - \\
\hline B 6 & - & $(+)$ & - \\
\hline \multicolumn{4}{|c|}{$\begin{array}{l}\text { * Degeneration and cell death. } \\
\text { † Irregular rates of mortality. } \\
\text { \$ Various degrees of cytoplasmic granulation. } \\
\& \text { Regular rates of mortality. }\end{array}$} \\
\hline
\end{tabular}

Cultural characteristics of B strains and Mycoplasma granularum. Five out of six B strains were isolated directly on PPLO agar. One strain, B6, had to be enriched in PPLO broth prior to cultivation on PPLO agar. The growth of B strains and Mycoplasma granularum was not stimulated by satellite cultivation but was dependent upon the presence of serum. The viable cell count gave 2 to $7 \times 10^{7}$ colony-forming units per $0.1 \mathrm{ml}$. of 2-day-old PPLO broth cultures. The colonies were embedded in the agar. Most colonies were of the 'fried egg' type, although the colonies of one strain, $\mathrm{B} 6$, differed from those of the other strains by having a structure similar to that described for $M$. pneumoniae (Channock, Hayflick \& Barile, 1962). The size of the organisms in the colonies of B strains was approximately $0 \cdot 2 \mu$.

One of the B strains, в 3, was shown to be strongly cytopathogenic for cultures of calf kidney cells and regularly lethal for chick embryos. Acid production was observed in cell cultures inoculated with this strain. The other B strains and Mycoplasma granularum have been passaged 3 times in eggs but proved to be non-lethal for chick embryos. Cell cultures inoculated with these strains showed various degrees of a cytoplasmic granulation. 
On the basis of some cultural properties indicated in Table 1, the porcine Mycoplasma strains can be differentiated into two groups, one represented by $M y c o$ plasma hyorhinis and the other by $\boldsymbol{M}$. granularum.

Differentiation by gel diffusion. In preliminary experiments, unabsorbed antisera and antisera absorbed with the PPLO broth medium were tested with this medium as antigen. Some unabsorbed sera gave one precipitation line with PPLO broth but most unabsorbed sera as well as all absorbed sera did not react with this medium. The results of cross-testing eleven absorbed antisera against suspensions of 12 strains are shown in Table 2. According to these results a close relationship exists between Mycoplasma hyorhinis and the SEP strains. The precipitation lines showed reactions of identity. SEP strains are thus identified as strains of $M$. hyorhinis. No cross-reactions were found between the group of strains represented by $M$. hyorhinis and the $\mathrm{B}$ strains including $\boldsymbol{M}$. granularum.

\section{Table 2. Differentiation of porcine Mycoplasma strains by gel diffusion}

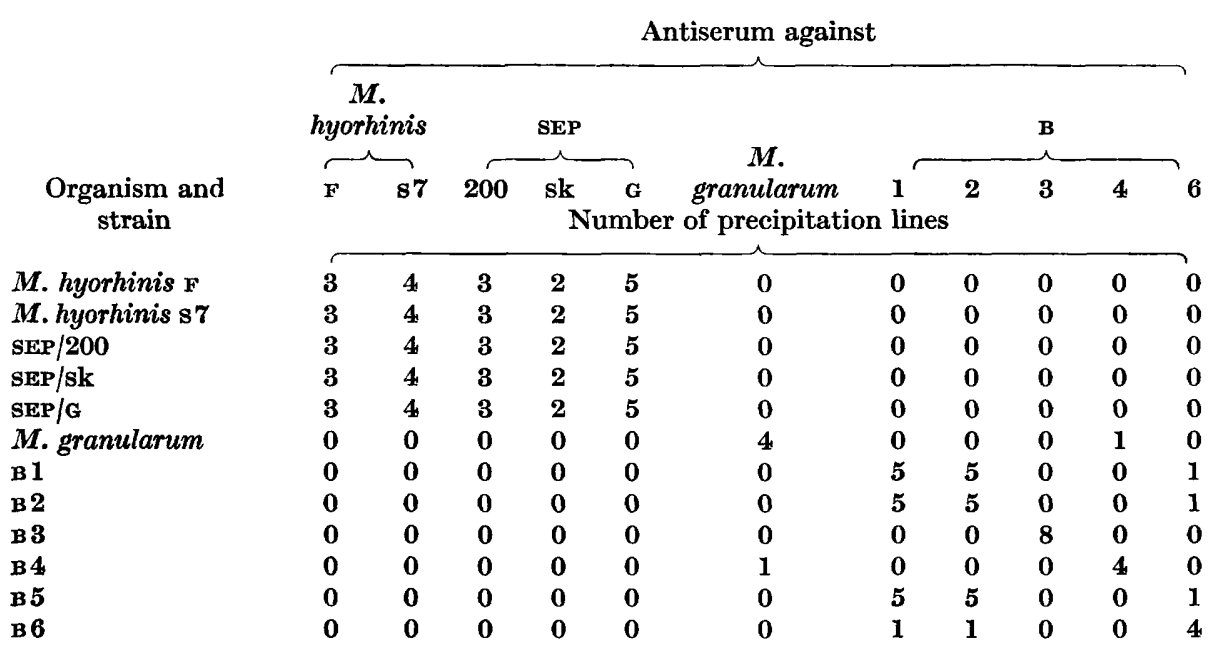

Within the group comprising the B strains and Mycoplasma granularum, a reci-

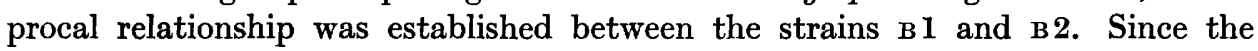
antigen of the в 5 strain was precipitated by antisera to strains в 1 and в 2, these three strains were considered to belong to the same serotype. Reciprocal crossreactions of low order were noted between $M$. granularum and strain $\mathrm{B} 4$ as well as between strain в 6 and the strains в 1, в 2 and в 5 . With heterologous antisera one precipitation line was formed by these strains whereas homologous antisera gave with them four or five lines (Table 2). No cross-reactions were observed between strain в 3 and the other strains although в 3 produced at least 8 lines when tested against homologous antiserum. According to these results the strains of the present study fall into six serotypes (Table 3).

Differentiation by growth inhibition. Antisera used in gel diffusion tests were also examined in growth inhibition tests. The results are summarized in Table 4. Antisera to SEP strains and strains of Mycoplasma hyorhinis inhibited the growth of the strain F of $M$. hyorhinis but had no inhibitory effect on the s7 strain of $M$. 
hyorhinis. All SEP strains were inhibited by homologous antisera and two of them also by certain heterologous antisera. The reciprocal relationship between SEP strains and strains of $M$. hyorhinis as shown by gel diffusion could thus not be demonstrated by growth inhibition. Concerning the B strains and $\boldsymbol{M}$. granularum, the specific reactions seen in gel diffusion tests were also observed in growth inhibition tests (Table 4). A close relationship was established between strains B 1, B 2, and в 5. The other three в strains and $M$. granularum were inhibited only by homologous antisera. Cross-reactions of low order seen between some strains in gel diffusion tests were not observed in growth inhibition tests.

Table 3. Serotypes of porcine Mycoplasma strains as shown by gel diffusion

\begin{tabular}{cl} 
Serotype & \multicolumn{1}{c}{$\begin{array}{c}\text { Organism and } \\
\text { strain }\end{array}$} \\
1 & M. hyorhinis: F, s7; \\
SEP: 200, sk, G & M. granularum \\
$\mathbf{3}$ & B3 \\
4 & B4 \\
5 & B1, B2, B5 \\
6 & B 6
\end{tabular}

Table 4. Differentiation of porcine Mycoplasma strains by growth inhibition

Antiserum against

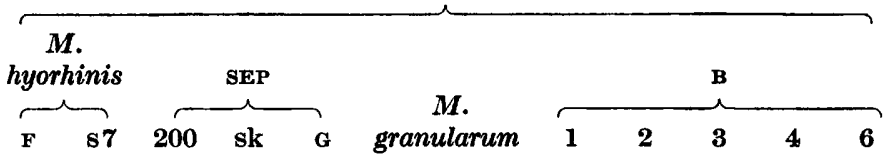

Strain

M. hyorhinis $\mathbf{F}$
M. hyorhinis $\mathbf{~ 7 7}$
SEP/200
SEP/sk
SEP/G
M. granularum
B1
B2
B3
B4
B5
B6

Zone of inhibition in millimeters

\begin{tabular}{|c|c|c|c|c|c|c|c|c|c|c|}
\hline 5 & 3 & 4 & 3 & 4.5 & - & - & - & - & - & - \\
\hline- & - & - & - & - & - & - & - & - & - & - \\
\hline 2.5 & $1 \cdot 5$ & 3 & - & $2 \cdot 5$ & - & - & - & - & - & - \\
\hline- & - & - & 2 & - & - & - & - & - & - & - \\
\hline 2 & - & 2 & - & 2 & - & - & - & - & - & - \\
\hline- & - & - & - & - & 5 & - & - & - & - & - \\
\hline- & - & - & - & - & - & 5 & 3 & - & - & - \\
\hline- & - & - & - & - & - & 5 & $\mathbf{3}$ & - & - & - \\
\hline- & - & - & - & - & - & - & - & $\mathbf{3 . 5}$ & - & - \\
\hline- & - & - & - & - & - & - & - & - & 4 & - \\
\hline- & - & - & - & - & - & $\mathbf{5}$ & 3 & - & - & - \\
\hline- & - & - & - & - & - & - & - & - & - & 5 \\
\hline
\end{tabular}

\section{DISCUSSION}

The porcine Mycoplasma strains isolated in this country could be divided at isolation into two groups. One group comprised isolates which originally grew better in cultures of animal cells and the yolk sac of chick embryos than on PPLO media unless satellite growth was employed. Isolates with such properties were encountered in more than $60 \%$ of pigs suffering from enzootic pneumonia or atrophic rhinitis (Bakos \& Dinter, 1963). These isolates were provisionally called 'sEP strains'.

The present study was carried out using SEP strains well-adapted to growth on 
PPLO media but these strains retained the properties of being cytopathogenic, irregularly lethal for chick embryos and promoted by satellite growth. It is interesting that they were shown to be closely related to Mycoplasma hyorhinis, originally isolated from pigs with atrophic rhinitis (Switzer, 1955) but considered to be a secondary invader (Switzer, 1964).

The strains of the other group were provisionally called в strains. The growth of these strains was dependent upon the presence of serum in the medium but was not stimulated by animal or bacterial cells as shown for SEP strains and Mycoplasma hyorhinis. $M$. granularum showed similar properties to the B strains. The existence of two different species, $M$. granularum and $M$. hyorhinis, was first established when porcine Mycoplasma strains were compared by indirect haemagglutination (Ross \& Switzer, 1963).

In the present study serological differentiation was carried out by gel diffusion and growth inhibition. These tests were found reliable for differentiation of human Mycoplasma strains (Taylor-Robinson et al. 1963, 1964). The gel diffusion tests showed that strains of Mycoplasma hyorhinis and SEP strains are antigenically identical. The growth inhibition test, on the other hand, proved to be unsatisfactory when applied for identification of $M$. hyorhinis. Antisera effective against one strain were less effective or ineffective against the other; one strain was not inhibited even by homologous antiserum. Klieneberger-Nobel (1962) and Huijsmans-Evers \& Ruys (1956) made similar observations when working with other Mycoplasma strains but no explanation is available for this phenomenon.

Both tests, the gel diffusion and growth inhibition, demonstrated that the $\mathbf{B}$ strains, including Mycoplasma granularum, are distinct from strains of $M$. hyorhinis but show great heterogeneity within the group. Only three out of six B strains belong to a single serotype whereas the other three в strains and $M$. granularum each represent separate serotypes. Although some heterologous strains showed cross-reactions in gel diffusion tests, the homologous reactions were sufficiently complex to identify the strains as separate serotypes. Similar observations were made with other Mycoplasma strains by Lemcke (1965).

Finally, a classification of porcine Mycoplasma strains must consider many more isolates than the small number studied here. Of special interest are the results of investigations carried out by the Cambridge group in this field. Goodwin \& Whittlestone (1964) cultivated a visible pleomorphic organism, the $J$ agent, from pigs with enzootic pneumonia, which was shown to induce a transmissible pneumonia in pigs. This agent is probably a Mycoplasma.

\section{REFERENCES}

Bakos, K. \& Dinter, Z. (1963). Das SEP-Agens. Proc. 17th World Vet. Congr. 1, 549.

Chanock, R. M., Hayflick, L. \& Barile, M. F. (1962). Growth on artificial medium of an agent associated with atypical pneumonia and its identification as a PPLO. Proc. natn. Acad. Sci., U.S.A. 48, 41.

Clyde, W. A., Jun. (1964). Mycoplasma species identification based upon growth inhibition by specific antisera. J. Immunol. 92, 958.

Dinter, Z., Wesslén, T. \& Lannek, N. (1957). Die Kultivierung des SEP-Agens im Dottersack des Bruteies. Zbl. VetMed. 4, 581.

Goodwin, R. F. W. \& Whittlestone, P. (1964). Production of enzootic pneumonia in pigs with a microorganism grown in media free from living cells. Vet. Rec. 76, 611. 
Huissmans-Evers, A. G. M. \& Ruys, A. C. (1956). Microorganisms of the pleuropneumonia group (Family of Mycoplasmataceae) in man. II. Serological identification and discussion of pathogenicity. Antonie van Leeurvenhoek, 22, 377.

Khieneberger-Nobel, E. (1962). Pleuropneumonia-Like Organisms (PPLO) Mycoplasmataceae. London and New York: Academic Press.

L'EcuYer, C., Switzer, W. P. \& RoberTs, E. D. (1961). Microbiologic survey of pneumonic and normal swine lungs. Am. J. vet. Res. 22, 1020.

LEMCKe, R. M. (1965). A serological comparison of various species of Mycoplasma by an agar gel double-diffusion technique. J. gen. Microbiol. 38, 91.

Morton, H. E., Smith, P. F. \& Leberman, P. R. (1949). Symbiotic growth of pleuropneumonia-like organisms with bacterial colonies. Proc. Soc. exp. Biol. Med. 72, 328.

Ross, R. F. \& SwItzer, W. P. (1963). Comparison of isolates of Mycoplasma hyorhinis by indirect hemagglutination. Am. J. vet. Res. 24, 622 .

SwITzER, W. P. (1955). Studies on infectious atrophic rhinitis. IV. Characterization of a pleuropneumonia-like organism isolated from the nasal cavities of swine. Am. J. vet. Res. 16, 540.

Switzer, W. P. (1964). Mycoplasmosis. In Diseases of Swine, 2nd ed, p. 498. Ed. by H. W. Dunne. Ames, Iowa, U.S.A.: The Iowa State University Press.

TAKÁtsy, Gy. (1955). The use of spiral loops in serological and virological micro-methods. Acta microbiol. Acad. sci. hung. 3, 191.

Taylor-Robinson, D., Canchola, J., Fox, H. \& Chanock, R. M. (1964). A newly identified oral mycoplasma $(M$. orale) and its relationship to other human mycoplasmas. Am. J. Hyg. 80, 135.

Taylor-Robinson, D., Somerson, N. L., Turner, H. C. \& Chanock, R. M. (1963). Serological relationships among human mycoplasmas as shown by complement-fixation and gel diffusion. J. Bact. 85, 1261.

WADSWORTH, C. (1957). A slide microtechnique for the analysis of immune precipitates in gel. Int. Archs Allergy appl. Immun. 10, 355.

Wesslén, T. \& LANNEK, N. (1954). The isolation and cultivation in tissue culture of a cytopathogenic agent from pigs with enzootic pneumonia (so-called virus pneumonia). Nord. VetMed. 6, 481. 